\title{
Von der Kosmetik zur ästhetischen Dermatologie
}

\section{From Cosmetics to Aesthetic Dermatology}

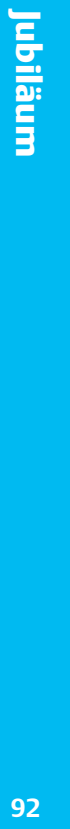

\section{Einleitung}

Ähnlich wie in anderen Fachgebieten haben sich die Fortschritte in der ästhetischen Dermatologie seit den 70er-Jahren rasant beschleunigt. Dieser Zweig der Dermatologie firmiert unter verschiedenen Namen, wie z.B. dermatologische Kosmetik, ästhetische Dermatologie, kosmetische Dermatologie etc.. Fast immer kommt in der Namensgebung dieses noch nicht einheitlich benannten Zweiges der Dermatologie das Wort „Kosmetik“, abgeleitet aus dem Griechischen: Kosmeté, Kunst des Schmückens, vor. Er beschäftigt sich mit der Korrektur von Normabweichungen oder Alterungsprozessen, die den Patienten mit Leidens-

\section{Abstract}

The enormous progress made within aesthetic dermatology since the beginning of the nineties has contributed to the innumerable modalities to treat the aging skin and sun-damaged skin like wrinkles, lentigines or scars.

In the field of tissue augmentation countless substances and techniques have been developed. The development of laser technology has broadened the spectrum of indications within dermatology for laser surgery. The refined methods for skin resurfacing have reduced their risks considerably during the last 10 years. A completely new approach in wrinkle treatment was established by introducing Botulinum Toxin A into aesthetic dermatology.

The development of aesthetic dermatology during the last 30 years is reviewed. druck belasten und zu einer Beeinträchtigung des Selbstwertgefühls führen können.

Die Tendenz, Altersveränderungen als belastend und damit als zu korrigierenden Zustand zu werten, ist in unserer vom SPIEGEL als „Spaß- und Wohlstandsgesellschaft“ titulierten Gesellschaft ständig zunehmend. Auf die Nachfrage nach einer verjüngten und faltenfreien Haut werden mit Slogans wie „Altern ist eine vermeidbare Krankheit“ in „Dienstleistungspaketen“ Schönheits- und Verjüngungskuren angeboten. Die Ausweitung der kosmetischen Dermatologie ist damit Ausdruck und in den heutigen Medien Gegenstand unsere derzeitigen gesellschaftlichen Tendenzen. 
Die Zuwendung vor allem im letzten Jahrzehnt zu einer kosmetisch orientierten Dermatologie ist eine Entwicklung unseres Faches, die von manchen [10] auch als Gefährdung angesehen wird, wenn die Motivation für das Interesse an „kosmetischen“ Behandlungsmodalitäten nur ökonomisch untermauert ist. Mit einer kommerziellen Denkweise verharren wir jedoch in der jahrhundertelangen Tradition, in der kosmetische Prozeduren der Scharlatanerie und der Alchemie nahe standen im Gegensatz zu den rein medizinischen Wissenschaften. Aus diesem Denken heraus wurde auch Gaspare Tagliacozzi, der vor über 400 Jahren die erste Nasenkorrektur beschrieben hatte, noch von der Kirche aus christlich geweihter Erde exhumiert und an einem unbekannten Ort verscharrt, weil er sich mit dem Eingriff in das von Gott gegebene Bild des Menschen versündigt hatte. Erst 1958 erklärte Papst Pius XII endlich, dass die ästhetische Chirurgie nicht im Widerspruch zum Willen Gottes steht.

40 Jahre später altert eine Generation mit einem Zuwachs an Lebensdauer, die zugleich im historischen Vergleich ungewöhnlich vermögend ist. Sie fordert immer mehr Dermatologen mit ihren Wünschen nach Faltenglättung, Entfernung von Altersflecken und unerwünschten Haaren, Absaugung von Fettpolstern etc. Dieser Bedarf an kosmetischen Leistungen einer breiten Bevölkerungsschicht ist eine Chance und eine Herausforderung für die moderne Dermatologie, die sich diesen neuen Aufgaben nur zögerlich stellt. In der von Scholz [12] 1999 veröffentlichten „Geschichte der Dermatologie in Deutschland“ kommt die kosmetische oder ästhetische Dermatologie nur im Rückblick auf die Jahrhundertwende zum 20. Jahrhundert bis in die 20er-Jahre vor. In dem Kapitel „Tendenzen in der Dermatologie in der BRD“ wird Schreus Einsatz für die ästhetische Medizin als ein Spezifikum des versuchten geistigen Neubeginns der 50er-Jahre abge$\tan$.

Zum ersten Mal bildete sich 1996 eine Arbeitsgemeinschaft für ästhetische Dermatologie und Kosmetologie, aber richtig hoffähig im wissenschaftlichen Sinne wurde die kosmetische Dermatologie erst 1999, als sich innerhalb der DDG die Arbeitsgruppe „ästhetisch korrektive und operative Dermatologie“ bildete.

Diese Arbeitsgruppe hat u. a. die Aufgabe, Leitlinien für die diversen Behandlungsmethoden der kosmetischen Dermatologie zu erarbeiten.

Hat sich die "kosmetische Dermatologie“ inzwischen etabliert? In den aktuellen Lehrbüchern der Dermatologie wird sie immer noch totgeschwiegen. Nur zögerlich tauchen seit Anfang der 90er-Jahre Publikationen über kosmetische Themen in den deutschsprachigen dermatologischen Fachzeitschriften auf, wohingegen sie in den pilzartig aus dem Boden geschossenen sog. kosmetischen Fachzeitschriften explosionsartig zugenommen haben. Mit der 2004 erschienenen „Kosmetischen Dermatologie“, von Worret und Gehring herausgegeben, und mit einem Geleitwort von Braun-Falco versehen, der schon früh die ästhetische Dermatologie förderte, scheint nun endlich der Durchbruch im deutschsprachigen dermatologischen Schrifttum gelungen.

Rückblickend waren die Möglichkeiten innerhalb einer kosmetischen Dermatologie in den 70er-Jahren noch sehr begrenzt. Sie musste sich im Wesentlichen auf die Behandlung von Besenrei- sern, Aknenarben, Alters-, und Pigmentflecken beschränken, wobei das von Schreus 1949 eingeführte hochtourige Schleifen neben Peellösungen verwendet wurden. Zur Faltenbehandlung stand nur Silikonöl zur Verfügung, meist obskurer Herkunft und mit Nebenwirkungen in Form von Granulomen behaftet. In den 70er-Jahren zogen erstmals Kosmetikerinnen in deutsche Universitätskliniken ein, abgesandt von einem Aknepräparatehersteller, die sog. „Aknetoiletten“ bei Aknepatienten durchführten. Seit Beginn der 80er-Jahre hat sich das Spektrum der Behandlungsmodalitäten in der kosmetischen Dermatologie derart vergrößert, so dass hier nur kursorisch der Werdegang der Wichtigsten skizziert werden kann.

\section{Entwicklung der Fülllmaterialien}

1981 begann die Ära der „Bio-Fillers“, die zur Gewebeaugmentation eingesetzt werden. Bei der Faltenbehandlung mit Füllmaterialien werden unterschiedliche gewebsverträgliche Substanzen in die Dermis oder ins Subkutangewebe injiziert und damit eine Gewebeaugmentation erreicht, die Falten verschwinden lässt. Unterschieden werden resorbierbare, nicht permanente Filler und nicht abbaubare, permanente Füllmaterialien, die seit Anfang der 90er-Jahre zur Faltenbehandlung zur Verfügung stehen. Dabei werden verschiedene Kanülen und Injektionstechniken verwendet. Inzwischen sind heute über 70 verschiedene Materialien zur Gewebeaugmentation im Handel (Tab.1-6). Um einen Überblick über die Entwicklung der Füllmaterialien zur Faltenbehandlung zu geben, muss ich mich hier auf einige wenige Substanzen beschränken, die sich im Laufe der Jahre bewährt haben.

Das weltweit am häufigsten verwendete Füllmaterial ist zur Zeit telopeptidarmes, hochgereinigtes, pepsinlösliches $35 \mathrm{mg} / \mathrm{ml}$ bovines Kollagen, das seit den frühen 70er-Jahren an der Stanford University, USA, entwickelt worden war und 1977 erstmals beim Menschen zur Behandlung von Aknenarben, subkutaner Atrophie und Falten verwendet wurde mit 50-85\% Korrektur und mit einer Langzeitwirkung von 3-12 Monaten. Dieses im Juli 1981 als Zyderm I ${ }^{\circledR}$ auf den Markt gebrachte Collagen wird mit einer 30 gauge Kanüle für die Behandlung vor allem von Falten verwendet. Hierbei muss, um eine mögliche Sensibilisierung auszuschließen, ein Hauttest 4 Wochen vor der Behandlung erfolgen. 1983 veröffentlichte Arnold Klein [6] nach $2 \frac{1}{2} 2$ Jahren klinischer Anwendung seine Erfahrungen mit mehr als $1000 \mathrm{~Pa}-$ tienten. Er propagierte die Überkorrektur des zu korrigierenden Defektes, Injektion in die Dermis und die serielle MikropunkturTechnik. Es folgten 1983 Zyderm II ${ }^{\circledR}$ mit $65 \mathrm{mg} / \mathrm{ml}$ bovinem Collagen und Zyplast ${ }^{\circledR}$ mit Glutaraldehyd vernetzt, das den Abbau des Kollagens im Gewebe verlangsamen sollte. Bis Mitte der 90er-Jahre hat Collagen als Füllmaterial den Markt in Deutschland beherrscht.

Nach der Markteinführung von Collagen folgte 1984 ein weiteres Füllmaterial, Fibrel $^{\circledR}$, ein lyophilisiertes Gelatine-Matrix-Implant aus Schweinehaut gewonnen mit Epsilon-Aminocapronsäure versetzt und mit patienteneigenem Serum gemischt. Millican [9] hat 1987 eine multizentrische Studie über die klinische Anwendung von Fibrel veröffentlicht. Nach der Genehmigung durch das FDA für Narben 1988 und für Falten 1990 war das ne- 
Tab. 1 Collagenpräparate

\section{Resorbierbare injizierbare Füllmaterialien Collagenpräparate}

\begin{tabular}{ll}
\hline Zyderm I & \\
\hline Zyderm II $^{\circledR}$ & Endoplast $^{\circledR}$ \\
\hline Zyplast $^{\circledR}$ & Autologen $^{\circledR}$ \\
\hline Resoplast $^{\circledR}$ & Cymetral $^{\circledR}$ \\
\hline Koken $^{\circledR}$ & DermiCol $^{\circledR}$ \\
\hline Atellokollagen $^{\circledR}$ & Dermalogen $^{\circledR}$ \\
\hline Zyplast $^{\circledR}$ & Isolagen $^{\circledR}$ \\
\hline Fibrel $^{\circledR}$ & ${\text { Cosmoderm } 1,2^{\circledR}}^{\circledR}$ Permacol Injection $^{\circledR}$ \\
\hline
\end{tabular}

Tab. 2 Hyaluronsäurepräparate

\section{Resorbierbare injizierbare Füllmaterialien}

Hyaluronsäurepräparate

\begin{tabular}{|c|c|}
\hline Hylaform Fine Lines ${ }^{\circledR}$ & Hyaluderm $^{\circledR}$ \\
\hline Hylaform ${ }^{\circledR}$ & Hyal-System ${ }^{\circledR}$ \\
\hline Hylaform Plus ${ }^{\circledR}$ & Juvederm $18,24,30^{\circledR}$ \\
\hline $\mathrm{Soft}^{\circledR}$ & Matridur ${ }^{\circledR}$ \\
\hline Restylane ${ }^{\circledR}$ & Matridex ${ }^{\circledR}$ \\
\hline Perlane $^{\circledR}$ & Rofilan $^{\circledR}$ \\
\hline Viscontour ${ }^{\circledR}$ & Reviderm Intra ${ }^{\circledR}$ \\
\hline Ac-Hyal ${ }^{\circledR}$ & Hyacell ${ }^{\circledR}$ \\
\hline \multirow[t]{2}{*}{ Hyal 2000 Injection } & Macrolane ${ }^{\circledR}$ \\
\hline & Mac Dermol ${ }^{\circledR}$ \\
\hline
\end{tabular}

Tab. 3 Resorbierbare injizierbare Füllmaterialien

Weitere resorbierbare injizierbare Füllmaterialien

\begin{tabular}{ll}
\hline Profill $^{\circledR}$ & Hyruan Injection $^{\circledR}$ \\
\hline Hyaldex $^{\circledR}$ & Juvederm 18,24 \\
\hline New Fill & Reviderm $^{\circledR}$ \\
\hline Bioinblue $^{\top M}$ & Radiance $^{\circledR}$ \\
\hline Bioinblue “Lightline“ & Matridur $^{\circledR}$ \\
\hline Fascian $^{\circledR}$ & Matridex $^{\circledR}$ \\
\hline Autologus fat & Hylan SeS $^{\circledR}$ \\
\hline Plasmagel & Fibroquel $^{\circledR}$ \\
\hline
\end{tabular}

benwirkungsarme Präparat mit einer das bovine Collagen übertreffenden Langzeitwirkung auch in Deutschland erhältlich, ist aber seit Mitte der 90er-Jahre vom deutschen Markt verschwunden.

Seit 1996 sind quervernetzte Hyaluronsäurepräparate für den Vertrieb zur Faltenbehandlung in Europa zugelassen. Hyaluronsäure ist ein Polysaccharid und ist bei allen Spezies und Gewebetypen identisch. Es zeichnet sich durch eine hohe Wasserbindungskapazität aus. Hyaluronsäurepräparate haben den Vorteil, dass kein Test vor der Behandlung durchgeführt werden muss
Tab. 4 Synthetische nicht-resorbierbare Füllmaterialien

Synthetische nicht resorbierbare injizierbare Füllmaterialien

\begin{tabular}{|ll|}
\hline Medical grade silicone & Aquamid $^{\circledR}$ \\
\hline PMS $350^{\circledR}$ & MetaCril $^{\circledR}$ \\
\hline Bioplastique $^{\circledR}$ & Bio-Alcamid $^{\circledR}$ \\
\hline Artecoll $^{\circledR}$ & Evolution $^{\circledR}$ \\
\hline DermaLive & Arteplast $^{\circledR}$ \\
\hline DermaDeep & \\
\hline Outline $^{\circledR}$ & Biocell Ultravital $^{\circledR}$ \\
\hline Argiform $^{\circledR}$ & Radiance $^{\circledR}$ \\
\hline Metrex $^{\circledR}$ & Bioformacryl $^{\circledR}$ \\
\hline
\end{tabular}

Tab. 5 Resorbierbare Festimplantate

Resorbierbare Festimplantate

AlloDerm ${ }^{\circledR} \quad$ Tutoplast $^{\circledR}$

Tab. 6 Nicht resorbierbare Festimplantate

\section{Nicht resorbierbare Festimplantate}

\begin{tabular}{|ll}
\hline Fil d'or $^{\circledR}$ & Soft Form $^{\circledR}$ \\
\hline Gore Tex $^{\circledR}$ & Advanta $^{\circledR}$ \\
Aptos $^{\circledR}$ & Medpore $^{\circledR}$ \\
\hline
\end{tabular}

wegen der fehlenden Antigenität. Es kann sofort mit der Behandlung begonnen werden. Maßgeschneiderte Moleküle ermöglichen die Anwendung in den verschiedenen Gesichtsarealen entsprechend der Dermisdicke und der Faltentiefe. Die handelsüblichen Hyaluronsäurepräparate haben sich in den letzten 2 Jahren vervielfacht. Derzeit sind ca. 20 verschiedene Hyaluronsäurepräparate (Tab. 2) auf dem deutschen Markt, die sich aufgrund ihrer biologischen Herkunft unterscheiden, indem sie aus Hahnenkämmen oder aus Bakterienkulturen gewonnen werden.

Die Erfahrung von fast 10 Jahren hat gezeigt, dass die Langzeitwirkung ungefähr derjenigen entspricht, die mit Collagenpräparaten erreicht werden kann. Ein neuerer Trend seit 2001 ist die großflächige Einspritzung der Haut mit nicht quervernetzter, nativer Hyaluronsäure zur Behandlung am Hals, Decolleté und Handrücken, wobei durch fächerförmige Injektion in die obere Dermis Turgor und Elastizität der Haut erhöht werden, und sie somit ein frischeres und strafferes Aussehen erhält.

1999 wurde kristalline Polymilchsäure eingeführt, ein synthetisch hergestelltes Polymer, das bioresorbierbar und -abbaubar ist. Es liegt in dem Präparat NewFill ${ }^{\circledR}$ als Lyophilisat vor und bedarf keines Allergietests. NewFill eignet sich nicht nur zur Korrektur von Falten und Narben, sondern auch zur Volumenauffüllung und Gesichtskonturierung. Es führt durch Neubildung von 


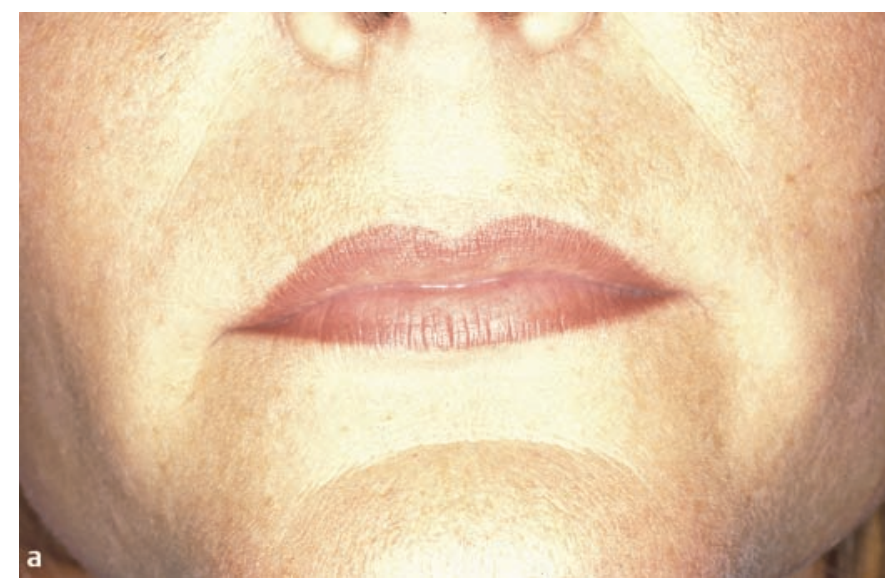

Abb. 1 Lippenkonturierung mit Dermalive ${ }^{\circledR}$. a vorher.

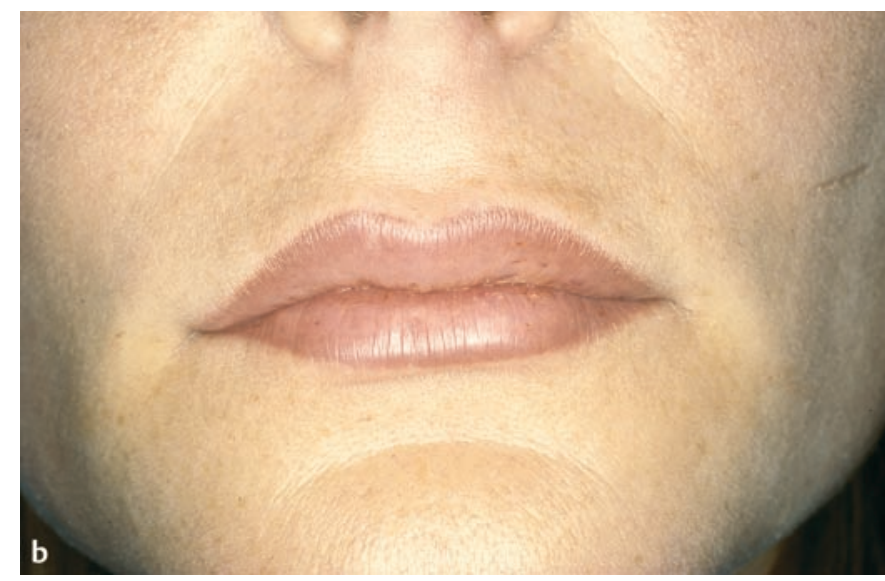

b 2 Jahre nach der Injektion mit Dermalive ${ }^{\circledR}$.
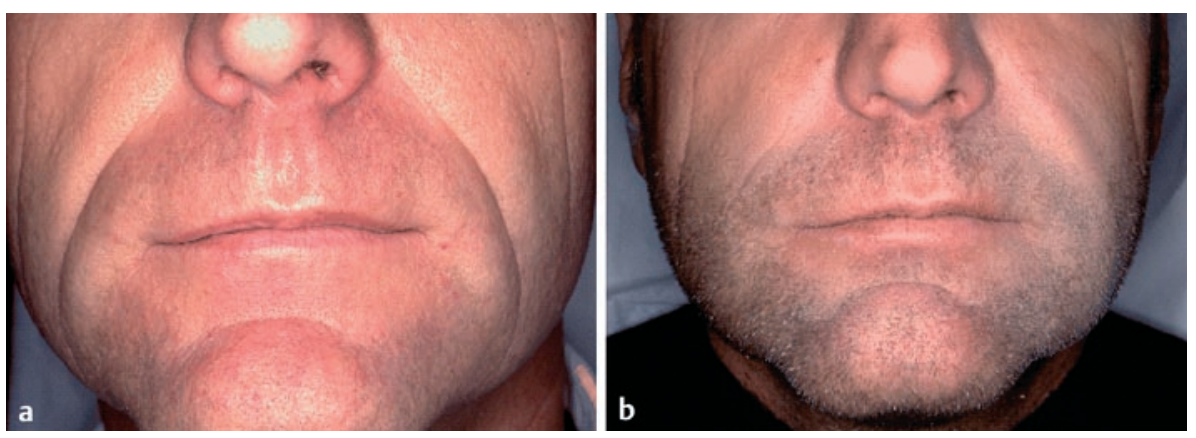

Abb. 2 Ergebnis nach Wire Scalpel-Behandlung (rechts).

Kollagenfasern zu einem Aufbau des Bindegewebes. Wie bei allen Bio-Fillern muss die Behandlung in gewissen Abständen wiederholt werden, um bessere Langzeitergebnisse zu erzielen. $\mathrm{Zu}$ Beginn der Markteinführung häuften sich Berichte über auftretende Knötchen, die nach einer höheren Verdünnung des Materials vor der Injektion mit Aqua dest. jetzt nur noch sporadisch auftreten. Die mit NewFill erhaltenen Resultate scheinen über 10 bis 20 Monate zu halten.

Nach den wegweisenden Arbeiten von Fischer und Fischer 1977 [4] gleichzeitig mit Illouz begann Ende der 70er-Jahre das moderne Zeitalter der Eigenfetttransplantation.

Fournier modifizierte die Technik und führte die Mikrolipoinjektion ein für die Behandlung von Gewebsdefekten und Falten. [5.] Als weiterer Vorreiter in der Methode des Lipofillings gelten Coleman und Amar, die multiple Fettgrafts in Muskel und Subkutis propagierten und damit bessere Langzeitergebnisse erzielten.

Die Methoden für Fettabsaugung zur Entfernung unerwünschten subkutanen Fettgewebes wurden seit 1990 zunehmend verfeinert durch Verringerung des Kanülendurchmessers und durch Austausch der spitzen Kanülenenden durch stumpfe. Heute wird vorwiegend mit Vibrationskanülen gearbeitet (Vibraliposuktion), die das Subkutangewebe geringer traumatisieren. Der revolutionäre Durchbruch kam, als Klein 1987 die Tumeszenslokalanästhesie [7] beschrieb, die heute mit elektrischen Pumpen durchgeführt wird, wobei Lidocain durch Prilocain ausgetauscht wurde [11]. Seitdem gilt die Fettabsaugung, die inzwi- schen mit speziellen Saugern und Auffangbehältern durchgeführt wird, als risikoarme Methode.

Als weiteres autologes Füllmaterial wird Plasmagel [8] seit Beginn 2000 zunehmend als sichere und billigere Alternative zur Gewebeauffüllung verwendet. Das vom Patienten gewonnene Plasma wird durch ein einfaches Erwärmungsverfahren geliert und unter die Falte gespritzt. Langzeitresultate stehen derzeit noch aus.

Artecoll ${ }^{\circledR}$ gelangte 1992 als eines der ersten synthetischen, nichtabbaubaren Füllmaterialien zur Gewebeaugmentation auf den deutschen Markt. Es enthält bovines Collagen mit 30 - $40 \mu \mathrm{m} \mathrm{Mi-}$ krosphären aus Polymethylmethacrylat (PMMA), die nicht phagozytiert werden können und somit ein besseres Langzeitergebnis bei der Faltenbehandlung bringen. Bei der Anwendung von synthetischen injizierbaren Füllmaterialien wird jedoch die Beherrschung einer speziellen Injektionstechnik vorausgesetzt, um die seltenen Nebenwirkungen von Fremdkörpergranulomen zu minimieren. Seit 1999 ist ein weiterer synthetischer Filler als Dermalive ${ }^{\circledR}$ und Dermadeep ${ }^{\circledR}$ im Handel, der aus Acrylhydrogelmikrosphären besteht mit Hyaluronsäure als Vehikel. Dermalive hat den Vorteilt, dass es durch sehr feine 30 gauge Kanülen injiziert werden und sich so besser im Gewebe verteilen kann (Abb. 1 u. 2). 

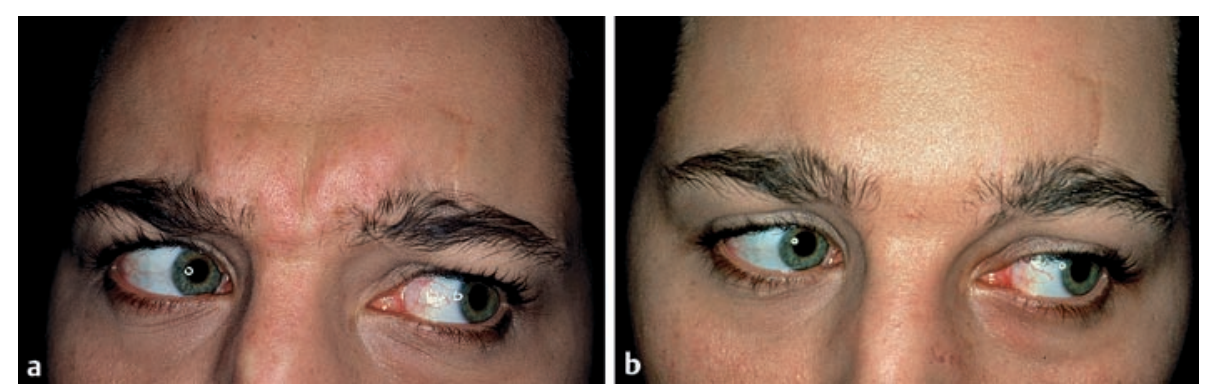

Abb. 3 Botulinum Toxin: vor und nach Behandlung.
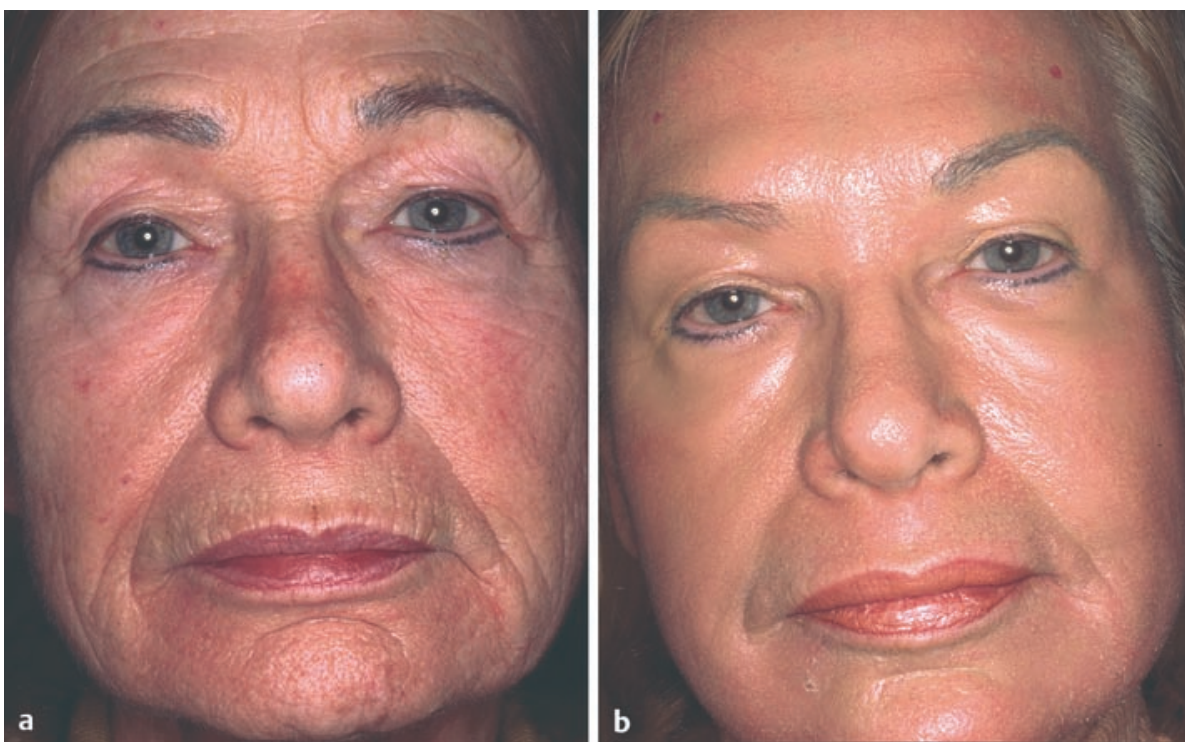

Abb. 4 Tiefes Chemical Peel vor und nach der Behandlung.

Gegen Ende der 90er Jahre zeichnete sich ab, wie die minimal-invasiven Methoden immer differenzierter eingesetzt wurden. Zum Anheben ptotischer Strukturen im präaurikulären, mandibulären und submandibulären Bereich hatte Sulamanidze Polypropylenfäden entwickelt, die mit konvergenten Haken versehen waren (Aptos ${ }^{\circledR}$-Fäden) [14], die subkutan in bestimmten Traktionslinien eingeführt werden. Bei jüngeren Patienten und in ausgesuchten Fällen konnte ein guter Liftingeffekt erreicht werden, die Aptos-Fäden können jedoch ein operatives Lift nicht ersetzen. Vom gleichen Autor wurde 1999 das „Wire Scalpel“ beschrieben, ein Stahlfaden, mit dessen Hilfe Falten und Narben

unterminiert werden und somit Fibrosierungen und in die Haut inserierende Muskelfasern durchtrennt werden können (Abb. 2)

\section{Botulinum Toxin in der ästhetischen Dermatologie}

Den wohl spektakulärsten Boom einer ästhetischen Behandlungsmaßnahme konnte die Behandlung von Gesichtsfalten mit Botulinum Toxin A verzeichnen, nachdem 1989 Carruthers und Carruthers [2] die Behandlung von Botulinum Toxin A für die kosmetische Anwendung beschrieben hatten. Botulinum Toxin A verhindert die Ausschüttung von Acetylcholin an der motorischen Endplatte und somit die Impulsübertragung vom Nerv auf die Muskelfaser. Dieser Effekt wird ausgenützt bei negativer Mimik mit Zornesfalten oder den hängenden Mundwinkeln, um durch Entspannung der entsprechenden Gesichtsmuskeln einen positiven Gesichtsausdruck zu erzielen und damit Falten zu glätten. Die selektive Lähmung der quergestreiften Muskulatur ist nach 4-6 Monaten wieder reversibel (Abb. 3u.4). Diese Methode zur Faltenglättung ist einfach in der Anwendung, effektiv und sicher. In Deutschland zunächst nur zögerlich aufgenommen, verbreitete sich die Methode seit Ende der 90er-Jahre sehr schnell und ist heute die am häufigsten angewandte Behandlungsmethode zur Glättung von Glabella-, Stirn- und Lachfalten. Der Effekt von Botulinum Toxin A, die Acetylcholinfreisetzung zu blockiern, wird auch zunehmend genutzt, um damit die fokale Hyperhidrosis zu behandeln. 
Bereits lange vor den 70er-Jahren waren verschiedene Peelsubstanzen bekannt, die die Haut bis zu unterschiedlichen Tiefen abschälen können und dadurch eine Regeneration von Epidermis und Dermis erreichen mit dem Ziel, Falten und Flecken zu entfernen. Baker und Gordon [1] hatten bereits 1961 die Erneuerung der Haut und Faltenbehandlung mit einer phenolhaltigen Lösung beschrieben, der Crotonöl, Septisol und Wasser beigemengt waren. Die Wirkung reicht bis ins Stratum reticulare der Dermis und bewirkt eine Neosynthese von Kollagen- und elastischen Fasern, wodurch das Volumen der Dermis wieder aufgebaut wird. Diese Lösung wird noch heute am häufigsten für tiefe Peels verwendet.

Auch die Wirkung der Trichloressigsäure (TCA) in verschiedenen Konzentrationen auf die Haut war schon lange bekannt. In den 80er-Jahren erlebten die Trichloressigsäurepeels zur Faltenbehandlung und Behandlung UV-geschädigter Haut eine Renaissance, als Stegmann [13] 1982 die Einwirktiefe von verschiedenen Peelsubstanzen anhand der nachfolgenden histologischen Veränderungen beschrieb und damit den Grundstein zur wissenschaftlichen Aufarbeitung der Chemical Peels legte. Die Einwirktiefe wurde kalkulierbarer. Seither wurden auch Kombinationen von TCA mit anderen Lösungen, z.B. Jessners Lösung und $\alpha$-Hydroxysäuren, angewandt als sog. Kombinationspeels, um einerseits die Wirkung der Trichloressigsäure zu verstärken und andererseits die möglichen Nebenwirkungen in Form von Narben und verzögerter Wundheilung zu minimieren.

In den 70er-Jahren hatte Van Scott [15] mit den Arbeiten über die $\alpha$-Hydroxysäuren begonnen, die den Anstoß gaben zum weltweiten Erfolg der Glykolsäurepeels seit Mitte der 80er-Jahre. Da die meisten Patienten heute keinen Arbeitsausfall nach einem Peeling zur Hautverjüngung hinnehmen möchten und der Wunsch nach „sanften Methoden“ größer geworden ist, haben sich die sog. „Lunch Time Peels“ durchgesetzt, die für oberflächliche Peels zur Verfügung stehen, und nach deren Anwendung klinisch keine Zeichen einer Schälung sichtbar werden. Die Wirkung ist abhängig von der verwendeten $\alpha$-Hydroxysäure, der Konzentration, dem pH-Wert der Lösung und der Verweildauer auf der Haut. Sie zeigt sich nach mehrmals wiederholter Behandlung in einer verbesserten Hautstruktur, Reduzierung von Komedonen und in der Aufhellung von Melasma. Das wachsende Interesse an den verschiedenen Peelmethoden spiegelt sich wider in der zunehmenden Kommerzialisierung der Peellösungen (Tab. 7), die früher nur vom Apotheker hergestellt wurden.

\section{Laserbehandlungen}

Mit der Faltenbehandlung durch Volumenauffüllung, mit der Behandlung der mimischen Muskulatur durch Botulinum Toxin A und mit der Hautverjüngung durch Peellösung konnten längst nicht alle Probleme im Bereich der kosmetischen Dermatologie gelöst werden. Man versuchte, Lösungen für störende Gefäßzeichnungen und störenden Haarwuchs im Bereich der Technologie zu finden.
Tab. 7 Auswahl aus z. Zt. handelsüblichen Chemical Peels

Auswahl aus den z. Zt. handelsüblichen ca. 45 Peel-Präparaten (Stand Januar 2004)

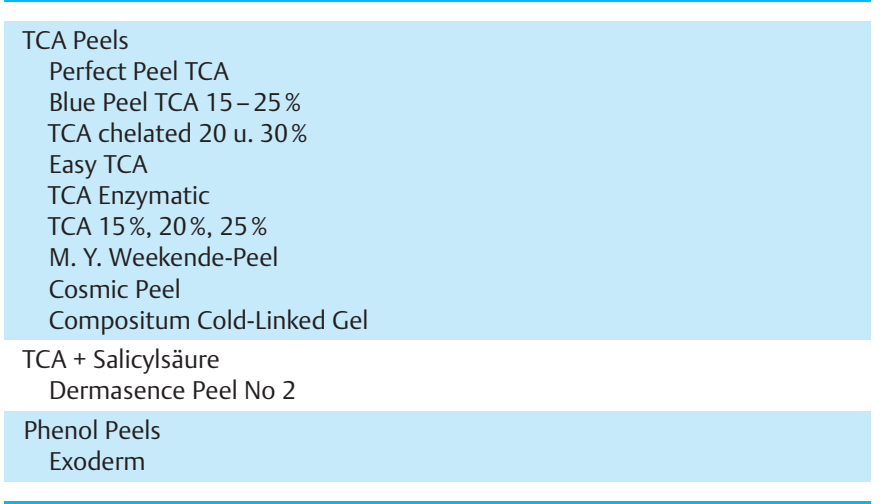

Die Entwicklung der modernen dermatologischen Lasertherapie ist Ausdruck des technologischen Fortschritts innerhalb der gesamten Medizin. Seit 1960 sind Lasergeräte schon in der Dermatologie bekannt, das Indikationsspektrum für Laseranwendungen hat sich aber erst anfangs der 90er-Jahre drastisch erweitert [3]. Nun war es möglich mit gütegeschalteten Festkörperlasern Pigmente in der Haut narbenfrei zu entfernen. Etwa zur gleichen Zeit wurden gepulste Farbstofflaser eingeführt, mit deren Hilfe feine Kapillaren rückstandslos zerstört werden konnten. Damit konnten Teleangiektasien im Gesicht wie an den Beinen behandelt werden. Ab 1994 konnte mit Hilfe von Scannern, die den fokussierten Laserstrahl kontinuierlich über eine Fläche führen, mit konventionellen $\mathrm{CO}_{2}$ Lasern die Haut flächig abgetragen werden. Parallel dazu wurde die Entwicklung der gepulsten $\mathrm{CO}_{2} \mathrm{La}-$ ser vorangetrieben. Diese technologischen Erneuerungen waren die Voraussetzung für ein großflächiges Skin-Resurfacing, mit dem auch heute noch Falten- und Narbenbehandlungen durchgeführt werden. Nun ging es Schlag auf Schlag in der weiteren technologischen Entwicklung. Es wurden Blitzlampen (Intense pulsed light sources, IPL) zunächst zur Koagulation von erweiterten Gefäßen entwickelt. 1995 kam der Durchbruch mit dem Photoderm, der zweiten Gerätegeneration dieser Blitzlampen. Dies löste auch die Entwicklung von Lasergeräten der IPL-Technologie zur Epilation aus. Die Entwicklung der ebenfalls abtragenden gepulsten Er:YAG-Laser erfolgte parallel zu der von gepulsten $\mathrm{CO}_{2}$-Lasern mit geringerer Nebenwirkungsrate, da die Abtragung in einem oberen Niveau der Haut erfolgt.

Ende der 90er-Jahre wurde nach der ersten Generation der IPLGeräte ein effizientes Gerät entwickelt, der Diodenlaser, der heute zum Standard für die Haarentfernung gehört.

Als neueste Entwicklung sind seit 2001 die Excimer-Laser anzusehen mit der Indikation zur Psoriasisbehandlung. Zur Zeit ist noch nicht abzusehen, inwieweit diese Technik auch ihren Platz bei Behandlungen im Bereich der kosmetischen Dermatologie finden wird. 
Lässt man die letzten 30 Jahre der kosmetischen Dermatologie Revue passieren, fällt auf, dass in der Dekade der 70er-Jahre zwar die Forschung intensiv auf allen Gebieten vorangetrieben wurde, der Niederschlag dieser Forschungen als Umsetzung im Bereich der klinischen Anwendung sich aber frühestens zu Beginn der 80er-Jahre bei den Füllmaterialien und der Lasertechnologie manifestiert. Ab Beginn der-90er Jahre ist die Entwicklung von immer neuen Technologien im Bereich der Lasergeräte nicht mehr zu bremsen. Dies gilt ebenso für die Füllmaterialien zur Gewebeaugmentation mit einer heute nicht mehr überschaubaren Menge an unterschiedlichen Substanzen. Als komplett neuer Behandlungsansatz bei der Faltenbehandlung in dem Jahrzehnt der neunziger Jahre gilt die Behandlung mit Botulinum Toxin A.

Manche bezweifeln, ob die fulminante Entwicklung von neuen Behandlungsmodalitäten der letzten 10 Jahre im Bereich der kosmetischen Dermatologie wissenschaftlichen Ansprüchen genügt. Orfanos spricht sogar von einer Spaltung und dem Auseinanderklaffen von klinischer und kosmetischer Dermatologie [10]. Die Ausweitung der kosmetischen Dermatologie durch die gesellschaftlichen Veränderungen zu Beginn des 21. Jahrhunderts erfordert dringend eine wissenschaftliche Auseinandersetzung mit diesem Teilgebiet der Dermatologie, um zu verhindern, dass eine Beschäftigung mit der kosmetischen Dermatologie als ein Abrutschen auf eine Ebene jenseits ärztlicher Ethik interpretiert wird.
${ }^{1}$ Baker TJ, Gordon HL. The ablation of rhytides by chemical means; a preliminary report. J Fla Med Assoc 1961; 48: 541

${ }^{2}$ Carruthers JA, Carruthers JDA. BOTOX treatment of glabellar frown lines and other facial wrinkles. J Dermatol Surg Oncol 1990; 10: 370 (abst)

${ }^{3}$ Drosner M. Anwendungen von Laser-und Blitzlicht. In: Worret WI, Gehring W (Hrsg). Kosmetische Dermatologie. Berlin, Heidelberg, New York: Springer, 2004

${ }^{4}$ Fischer A, Fischer GM. Revised Technique for cellulitis fat Reduction in riding breeches deformity. Bull Int Acad Cosm Surg 1977; 2: 40

${ }^{5}$ Fournier PF. Microlipoextraction et microlipoinjection. Rev chir Esthet Lang Franc 1985; 10: 36- 40

${ }^{6}$ Klein AW. Implantation techniques for injectable collagen. J Am Acad Dermatol 1983; 9: 224-228

${ }^{7}$ Klein JA. The tumescent technique for liposuction surgery. Am J Cosmetic Surg 1987; 4: 263 - 267

${ }^{8}$ Kraijcik R, Orentreich DS, Orentreich N. Plasmagel: A Novel Injectable Autologous Material for Soft Tissue Augmentation. J Aesth Derm and Cosm Surg 1999; 1: 109- 115

${ }^{9}$ Millican L, Rosen T, Monheit G. Treatment of depressed cutaneous scars with gelatine matrix implant: A multicenter study. J Am Acad Dermatol 1987; 16: $1155-1162$

10 Orfanos CE, Christophers E. Von der Dermatologie in die Kosmetik. Chance oder Risiko? Hautarzt 2002; 53: 1 -4

${ }^{11}$ Sattler G, Rapprich S, Hagedorn M. Tumeszens-Lokalanaesthesie-Untersuchung zur Pharmakokinetik von Prilocain. Z Hautkr 1997; 72: $522-525$

12 Scholz A. Geschichte der Dermatologie in Deutschland Berlin, Heidelberg: Springer, 1999

13 Stegman SJ. A comparative histologic study of the effects of three peeling agents and dermabrasion on normal and sun-damaged skin. Aesthet Plast Surg 1982; 6: $123-135$

${ }^{14}$ Sulamanidze MA, Fournier PF, Paikidzet TG, Sulamanidze GM. Removal of Soft Facial Tissue Ptosis with spezial Threads. Derm Surg 2002; 28: $367-371$

${ }^{15}$ Van Scott EJ, Yu RU. Hyperkeratinization, corneocyte cohesion and alpha hydroxyl acids. J Acad Dermatol 1984; 11: 876-879

${ }^{16}$ Walter C, Wiest LG. Gore-Tex: Eine Offensive bei der Faltenkorrektur. In: Tilgen W, Petzold D (Hrsg). Operative und konservative DermatoOnkologie. Berlin, Heidelberg: Springer, 1995 